Marquette University

e-Publications@Marquette

$1-1-2000$

\title{
On the Stabilizing Action of Protein Denaturants: Acetonitrile Effect on Stability of Lysozyme in Aqueous Solutions
}

Evgueni Kovriguine

Marquette University, evgueni.kovriguine@marquette.edu

Sergey A. Potekhin

Accepted version. Biophysical Chemistry, Vol. 83, No. 1 (January 2000): 45-59. DOI: (C) 2000 Elsevier Science B.V. Used with permission. 


\title{
Marquette University
}

\section{e-Publications@Marquette}

\section{Chemistry Faculty Research and Publications/College of Arts and Sciences}

This paper is NOT THE PUBLISHED VERSION; but the author's final, peer-reviewed manuscript. The published version may be accessed by following the link in the citation below.

Biophysical Chemistry, Vol. 83, No. 1 (January 2000): 45-59. DOI. This article is (C) Elsevier and permission has been granted for this version to appear in e-Publications@Marquette. Elsevier does not grant permission for this article to be further copied/distributed or hosted elsewhere without the express permission from Elsevier.

\section{On the stabilizing action of protein denaturants: acetonitrile effect on stability of lysozyme in aqueous solutions}

\author{
Evgenii L. Kovrigin
}

Department of Chemistry, Marquette University, Milwaukee, WI

Institute of Protein Research, Russian Academy of Sciences, Pushchino, Moscow 142292, Russia

Sergey A. Potekhin

Institute of Protein Research, Russian Academy of Sciences, Pushchino, Moscow 142292, Russia

\begin{abstract}
Stability of hen lysozyme in the presence of acetonitrile (MeCN) at different $\mathrm{pH}$ values of the medium was studied by scanning microcalorimetry with a special emphasis on determination of reliable values of the denaturational heat capacity change. It was found that the temperature of denaturation decreases on addition of $\mathrm{MeCN}$. However, the free energy extrapolation showed that below room temperature the thermodynamic stability increases at low concentrations of $\mathrm{MeCN}$ in spite of the general destabilizing effect at higher concentrations and temperatures. Charge-induced contribution to this stabilization was shown to be negligible (no $\mathrm{pH}$-dependence was found); therefore, the most probable cause for the phenomenon is an increase of hydrophobic interactions at low temperatures in aqueous solutions containing small amounts of the organic additive. The difference in preferential solvation of native and denatured states of lysozyme was calculated from the stabilization free energy data. It was found that the change in preferential solvation strongly depends on the
\end{abstract}


temperature in the water-rich region. At the higher $\mathrm{MeCN}$ content this dependence decreases until, at 0.06 mole fractions of $\mathrm{MeCN}$, the difference in the preferential solvation between native and denatured lysozyme becomes independent of the temperature over a range of $60 \mathrm{~K}$. The importance of taking into account nonideality of a mixed solution, when analyzing preferential solvation phenomena was emphasized.

\section{Keywords}

Protein stability, Preferential solvation, Lysozyme, Scanning calorimetry, Mixed solvents

\section{Introduction}

During recent decades the considerable interest in cryobiology was attracted to the apparent paradox that some organic substances, such as dimethyl sulfoxide, polyethylene glycol, ethanol, etc., could serve as cryoprotectants for proteins, yet could also induce protein denaturation at elevated temperatures. This subject was thoroughly addressed in some excellent reviews [1], [2]. They proposed that the most important factor responsible for such dual behavior is the temperature dependence of hydrophobic interactions of cosolvents and water with proteins. There are three groups of evidence available to illustrate this phenomenon. The first one comes from the very cryobiology, that certain substances of non-polar organic character can be toxic for cells and tissues at about, say, $0^{\circ} \mathrm{C}$ while they are perfectly working as cryoprotectants at $-30^{\circ} \mathrm{C}$ (see [1]). However, these observations cannot serve alone as a basis for hypothesis that denaturants can turn to stabilizers at low temperatures. There could be another reason to account for such behavior (at least in some cases), namely, a dramatic decrease in unfolding rate upon both adding non-polar components and lowering the temperature (i.e. see [3]).

The second group of evidence is based on spectroscopic measurements of the effects of organic co-solvents on the denaturation temperature. It was found that the denaturing ability of some additives decreases on lowering the temperature (different $\mathrm{pH}$ values of the medium were used to have denaturation transition in different temperature regions - [4], [5], [6]). The most convincing evidence that this stabilization effect is a real phenomenon was obtained by Fujita and Noda [7] for RNAse A-ethylene glycol-water system. In that work, the authors found that at higher $\mathrm{pH}$ values and, correspondingly, at higher temperatures, ethylene glycol imposed a destabilizing effect on the protein denaturation temperature while at lower temperatures (lower $\mathrm{pH}$ ) the protein was stabilized on addition of ethylene glycol (i.e. the denaturation temperature increased).

At last, there is a number of DSC works on various denaturing organic additives where authors, using the free energy extrapolations, supposed existence of the stabilizing effect at low temperatures (see, for example [7], [8], [9], [10], [11]). However, in all of them, except for ethylene glycol-RNAse system [11], this phenomenon was predicted to take place at rather low temperatures (about room temperature) while denaturational data were obtained in the temperature region $40-70^{\circ} \mathrm{C}$. Therefore, all these predictions were made using extrapolation of the stabilization free energy values to the temperature of interest. However, in most cases the heat capacity change $\Delta C_{p}$ was taken as temperature- and cosolvent-independent without actual measurements and, though the free energy estimates provide the most direct information about the protein stability, this neglect of probable changes in $\Delta C_{p}$ puts the conclusions on rather shaky grounds.

Therefore, it appeared necessary to study the effect of organic additives on protein thermodynamic stability at low temperatures with a special emphasis on obtaining reliable values of the heat capacity change upon unfolding. Having this quantity measured, one can safely use extrapolation procedures to examine additives' effect on protein stability in the low temperature region. This paper reports the results of such a study and it addresses the effect of acetonitrile (MeCN) (an organic solvent widely used in protein chemistry and enzymology) on hen egg lysozyme, a typical small globular protein, whose thermodynamics in aqueous solutions was well characterized. 


\section{Experimental}

\subsection{Chemicals and equipment}

Hen Egg White Lysozyme was obtained from Merck, Darmstadt and from Reakhim, Russia. The protein was additionally recrystallized from $4 \% \mathrm{NaCl}$ solution, extensively dialyzed against doubly distilled water, lyophilized and stored at $4^{\circ} \mathrm{C}$. Purity of the protein preparations was checked by SDS-gel electrophoresis which showed more than $95 \%$ homogeneity to the protein sample. No calorimetric difference between the protein preparations from the two suppliers (Merck and Reakhim) was observed. Acetonitrile (MeCN) was obtained from Serva (HPLC grade) and used without further purification. Water for experiments was doubly distilled.

Differential scanning microcalorimetry experiments were done using a SCAL 1 microcalorimeter (SCAL Co. Ltd., Pushchino, Russia) and a DASM-4A microcalorimeter (BioPribor, Pushchino, Russia). Protein samples for calorimetry were prepared by $12-16-\mathrm{h}$ dialysis at $4^{\circ} \mathrm{C}$. Typical protein concentrations were about $2 \mathrm{mg} / \mathrm{ml}$. Thermograms recorded in calorimetric experiments were processed to determine peak area and transition temperature (as a peak maximum) using the original software SCAL 2.0 (Scal Co. Ltd., Pushchino, Russia).

\subsection{Buffer solutions and $\mathrm{pH}$ measurements in a mixed solvent}

The buffer solutions for experiments were prepared to have $20 \mathrm{mM}$ glycine- $\mathrm{HCl}$ (for pH 2-3.6) or $20 \mathrm{mM}$ Sodium acetate-acetic acid (for $\mathrm{pH} 3.8-5.5$ ) using $0.1 \mathrm{M}$ stock solutions of the above buffer salts. The MeCN content was controlled at all stages of the experiment using refraction index measurements.

$\mathrm{pH}$ values of buffer solutions were adjusted with concentrated acetic acid or $\mathrm{HCl}$ containing the same amount of $\mathrm{MeCN}$ as in the buffer solution to prevent the change of $\mathrm{MeCN}$ concentration during $\mathrm{pH}$ adjustment. This procedure allowed us to prepare a series of solutions with a precisely constant MeCN content and different acidity. Apparent $\mathrm{pH}$ values of water-MeCN solutions were measured by a PHM82 Standard $\mathrm{pH}-\mathrm{meter}$ (Radiometer, Copenhagen) using a standard universal electrode.

Conversion of the apparent $\mathrm{pH}$ value $\left(\left(\left(_{(())}\right)\right.\right.$of an organic-water solution to the real one $\left(()_{(u()}\right)$ should involve two corrections: one is associated with the appearance of the additional potential at the surface of the reference electrode membrane; another one stems from the change of the proton activity coefficient $\gamma_{H}$ in the presence of MeCN [12], [13]. The influence of the added organic solvent upon $\gamma_{H}$ could be estimated using the Debye-Hückel equation with empirical constants. Unfortunately, the latter were unavailable for $\mathrm{MeCN}$-water solutions, nevertheless, in order to estimate the magnitude of the effect we calculated $\gamma$ ( for $60 \%$ DMSO, $40 \%$ methanol and $40 \%$ ethanol solutions in water (data from [13]). The corrections in the apparent $\mathrm{pH}$ values due to the solvent-induced change in activity coefficients appeared to be $0.007,0.019$ and $0.029 \mathrm{pH}$ units, respectively. These values are really small, so one could expect the effect of MeCN on the proton activity also to be negligible. Therefore, the proton activity coefficients $\gamma_{\text {( for }} \mathrm{MeCN}$-water solutions were assumed to be constant in this work.

The correction for the additional potential at the membrane of the reference electrode (at the surface of phase separation) could be found from the $\mathrm{pH}$ measurements at a constant real $\mathrm{pH}$ value. Assuming $\mathrm{\gamma}$ ( to be independent of the solvent composition, the constant concentration of strong acid could provide constant ( $($ (l( so any changes in apparent $\mathrm{pH}$ readings, to a good approximation, would correspond to the MeCN-induced additional potential [12], [13]. Thus we measured the ( («) values of $0.001 \mathrm{M} \mathrm{HCl}$ solutions with different $\mathrm{MeCN}$

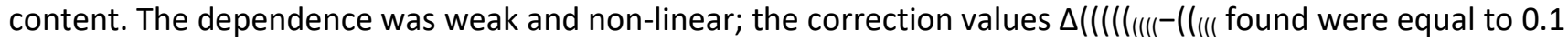
$\mathrm{pH}$ units for $x_{3}=0.037$ (mole fraction of $\mathrm{MeCN}$ ) and $0.13 \mathrm{pH}$ units for $x_{3}=0.075$. 


\subsection{Protein concentration measurements}

Protein concentrations in the solutions were determined by UV absorption at $281 \mathrm{~nm}$ using specific absorption $\varepsilon_{\|(I}=2.67$ o.u. $\left(\mathrm{mg} / \mathrm{ml}^{-1} \mathrm{~cm}^{-1}\right.$. Possible influence of MeCN upon UV-light absorption of lysozyme was shown to be negligible: small aliquots of the protein stock solution in water were added to aliquots of MeCN-water mixtures and the resulting absorption was measured against the reference solutions prepared by the same procedure without the protein; all measured values were about 2.67 within $5 \%$ error.

\subsection{Computational procedure for calculation of thermodynamic functions taking into account uncertainties of raw experimental data}

Experimental enthalpies and temperatures of transitions were fitted with a set of analytical equations to obtain numerical model the transition parameters as functions of solvent composition and $\mathrm{pH}$ value of the medium. Details on these equations are presented in Appendix A. Reliability of fitting results was determined as recommended by Johnson and Faunt [14] and Straume and Johnson [15], and the procedure employed was as follows.

1. Raw experimental data were fitted with chosen analytical equations by weighted Nelder-Mead simplex algorithm; 'perfect' (best-fit) values of temperatures and enthalpies for each experiment were calculated.

2. These 'perfect' data were perturbed using random Gaussian distributed noise; each data point (corresponding to the result of a single calorimetric experiment) was perturbed taking its individual uncertainty as a standard deviation for perturbation.

3. The perturbed data set was fitted using the same analytical equations.

4. Steps 2 and 3 were repeated 100 times collecting parameters from fitting runs.

5. These 100 sets of fitting parameters were used further in calculations of all thermodynamic functions of interest; the calculations were performed separately with each parameter set and the results were averaged. Estimation of the confidence interval for the result gave us sensitivity of the calculated functions to uncertainties in the initial experimental data.

\section{Results}

The DSC experiments were planned to determine $\Delta C_{p}$ values with maximal practically achievable precision. For this purpose, the $\mathrm{pH}$ values for solutions with the constant $\mathrm{MeCN}$ content were taken so that the transition temperature variation would be maximal: this allowed us to evaluate the derivative $\partial \Delta_{d} H / \partial T_{d}$ most accurately. Under all experimental conditions, lysozyme denaturation was highly reversible and shown to be fairly equilibrium: decreasing the scan rate from 1 to $0.1 \mathrm{~K} / \mathrm{min}$ did not significantly change the transition profiles. A slight decrease in the unfolding rate at the most acidic conditions and the highest MeCN content caused a shift of peak maxima down by 1-1.5 K when the scan rate was decreased 10-fold. Reversibility of the lysozyme unfolding was more than $95 \%$ as judged from the repeated scans. The above-mentioned made it possible to apply equilibrium thermodynamics for analysis of the data obtained. The raw experimental data are given in Table 1.

Table 1. Results of DSC experiments with hen lysozyme in aqueous acetonitrilea

\begin{tabular}{|l|c|c|l|l|l|l|l|c|}
\hline $\boldsymbol{X}_{\mathbf{3}}$ & $\mathbf{p} \boldsymbol{H}_{\text {real }}$ & $\boldsymbol{T}_{\mathrm{d}}(\mathbf{K})$ & $\boldsymbol{\Delta}_{\mathrm{d}} \boldsymbol{H}_{\text {cal }}(\mathbf{k J} / \mathbf{m o l})$ & $\mathbf{\pm} \boldsymbol{\delta} \boldsymbol{\Delta}_{\mathrm{d}} \boldsymbol{H}_{\text {cal }}$ & $\boldsymbol{\Delta}_{\mathrm{d}} \boldsymbol{H}_{\mathrm{vh}}(\mathbf{k J} / \mathbf{m o l})$ & $\mathbf{\pm} \boldsymbol{\delta} \boldsymbol{\Delta}_{\mathrm{d}} \boldsymbol{H}_{\mathrm{vh}}$ & $\boldsymbol{\Delta}_{\mathrm{d}} \boldsymbol{H}_{\mathrm{vh}} \boldsymbol{\Delta}_{\mathrm{d}} \boldsymbol{H}_{\mathrm{cal}}$ & $\mathbf{\pm} \boldsymbol{\delta}$ \\
\hline $\mathbf{0}$ & $\mathbf{4 . 5 4}$ & $\mathbf{3 5 0 . 7}$ & $\mathbf{4 8 9}$ & $\mathbf{5}$ & $\mathbf{5 2 0}$ & $\mathbf{4}$ & $\mathbf{1 . 0 6}$ & $\mathbf{0 . 0 2}$ \\
\hline 0 & 4.54 & 350.7 & 505 & 23 & 504 & 14 & 1.00 & 0.07 \\
\hline 0 & 4.54 & 350.7 & 495 & 17 & 502 & 10 & 1.01 & 0.05 \\
\hline 0 & 3.83 & 349.5 & 493 & 17 & 510 & 12 & 1.04 & 0.06 \\
\hline 0 & 3.83 & 349.5 & 490 & 17 & 506 & 17 & 1.03 & 0.07 \\
\hline 0 & 3.83 & 349.5 & 476 & 4 & 511 & 2 & 1.07 & 0.01 \\
\hline
\end{tabular}




\begin{tabular}{|l|l|l|l|l|l|l|l|l|}
\hline 0 & 3.55 & 350.7 & 494 & 3 & 503 & 2 & 1.01 & 0.01 \\
\hline 0 & 3.55 & 350.7 & 495 & 13 & 499 & 10 & 1.01 & 0.05 \\
\hline 0 & 3.00 & 347.4 & 474 & 38 & 482 & 24 & 1.0 & 0.1 \\
\hline 0 & 3.00 & 347.0 & 477 & 38 & 492 & 24 & 1.0 & 0.1 \\
\hline 0 & 2.67 & 342.3 & 465 & 24 & 464 & 15 & 1.00 & 0.08 \\
\hline 0 & 2.67 & 342.3 & 452 & 22 & 449 & 17 & 1.00 & 0.09 \\
\hline 0 & 2.37 & 336.6 & 449 & 24 & 417 & 15 & 0.9 & 0.1 \\
\hline 0 & 2.37 & 336.6 & 421 & 18 & 421 & 13 & 1.00 & 0.07 \\
\hline 0 & 1.88 & 325.1 & 308 & 20 & 363 & 9 & 1.18 & 0.08 \\
\hline 0 & 1.88 & 325.1 & 312 & 27 & 359 & 11 & 1.15 & 0.10 \\
\hline 0 & 1.88 & 325.2 & 352 & 20 & 355 & 11 & 1.01 & 0.09 \\
\hline 0 & 1.53 & 321.9 & 322 & 4 & 357 & 4 & 1.11 & 0.02 \\
\hline 0 & 1.53 & 322.1 & 338 & 10 & 348 & 8 & 1.03 & 0.05 \\
\hline 0.037 & 3.38 & 341.9 & 510 & 5 & 501 & 5 & 0.98 & 0.02 \\
\hline 0.037 & 3.38 & 341.9 & 490 & 25 & 515 & 15 & 1.05 & 0.08 \\
\hline 0.037 & 3.38 & 341.9 & 488 & 5 & 514 & 5 & 1.05 & 0.02 \\
\hline 0.037 & 3.02 & 337.4 & 476 & 8 & 486 & 6 & 1.02 & 0.03 \\
\hline 0.037 & 3.02 & 337.4 & 507 & 20 & 472 & 14 & 0.93 & 0.07 \\
\hline 0.037 & 2.60 & 331.4 & 444 & 11 & 457 & 8 & 1.03 & 0.04 \\
\hline 0.037 & 2.60 & 331.7 & 446 & 14 & 458 & 10 & 1.03 & 0.05 \\
\hline 0.075 & 3.40 & 329.9 & 436 & 9 & 460 & 8 & 1.06 & 0.04 \\
\hline 0.075 & 3.40 & 329.9 & 433 & 16 & 463 & 11 & 1.07 & 0.06 \\
\hline 0.075 & 3.40 & 330.0 & 451 & 15 & 451 & 9 & 1.00 & 0.05 \\
\hline 0.075 & 2.46 & 313.8 & 364 & 15 & 387 & 9 & 1.06 & 0.06 \\
\hline 0.075 & 2.46 & 313.7 & 363 & 16 & 383 & 9 & 1.06 & 0.06 \\
\hline 0.075 & 2.96 & 323.1 & 424 & 23 & 416 & 14 & 0.98 & 0.09 \\
\hline 0.075 & 2.96 & 323.4 & 393 & 14 & 438 & 7 & 1.12 & 0.05 \\
\hline & & & & & & & \\
\hline 0 & & & & 9 & & \\
\hline
\end{tabular}

a. Column headings are: $x_{3}$, the acetonitrile mole fraction; $\mathrm{pH}_{\text {real, }}$ the $\mathrm{pH}$ value of a mixed solution corrected the additional 'phase' potential (for details, see Section 2); $T_{\mathrm{d}}$, the temperature of a midpoint of protein denaturation transition (K); $\Delta_{\mathrm{d}} H_{\mathrm{cal}}$, the enthalpy of a denaturation transition, $(\mathrm{kJ} / \mathrm{mol}) ; \delta \Delta_{\mathrm{d}} H_{\mathrm{cal}}$, the absolute uncertainty of the calorimetric enthalpy value; $\Delta_{d} H_{v h}$, the effective enthalpy of a denaturation transition, van't Hoff enthalpy $(\mathrm{kJ} / \mathrm{mol}) ; \delta \Delta_{\mathrm{d}} H_{\mathrm{vh}}$, the absolute uncertainty of the effective enthalpy value; $\Delta_{\mathrm{d}} H_{\mathrm{vh}} / \Delta_{\mathrm{d}} H_{\mathrm{cal}}$, the van't Hoff criterion for the two-state mechanism of denaturation transitions; the last column, $\delta$, the uncertainty of the van't Hoff criterion value.

Fig. 1 represents the temperatures of denaturation transitions ( vs. the (( value of the medium at the three solution compositions. The data for solutions without $\mathrm{MeCN}$ are in excellent agreement with those reported by Privalov and Khechinashvili [16] for this protein. One can see that the shape of dependencies is similar for all three solution compositions: an addition of $\mathrm{MeCN}$ shifts the curves down with no significant changes in their slope. The lines in the figure are the best fits by the polynomial model. Details on the temperature data fitting are given in Appendix A. 


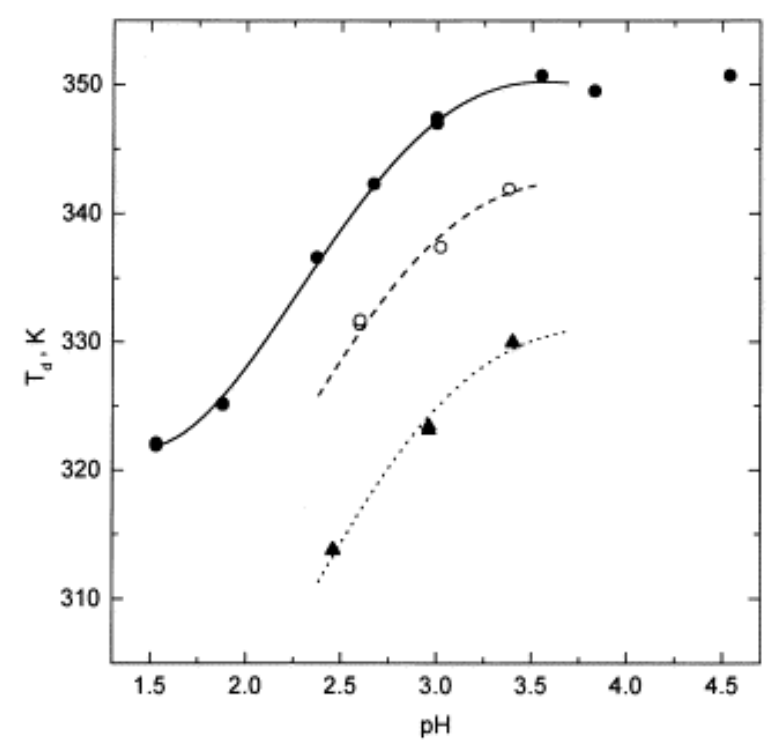

Fig. 1. The lysozyme denaturation temperatures plotted vs. the $\mathrm{pH}$ values of the solution. The MeCN content is indicated by: black circles, $x_{3}=0$; white circles, 0.037 ; black triangles, 0.075 . The lines are best-fit results for the $\mathrm{pH}$ range of interest.

Fig. 2 shows the heat absorbed on unfolding transitions plotted vs. corresponding transition temperatures. Lines in this plot show the best fit results (for details see Appendix A). The slopes of these lines appear to be statistically indistinguishable, so we can regard $\Delta$ (, to be independent of the MeCN concentration in our experiments.

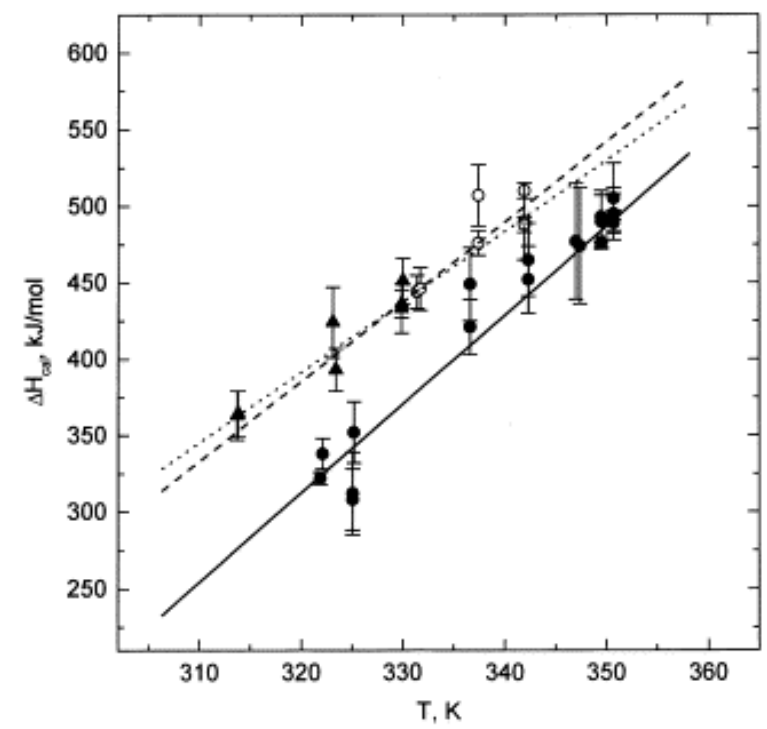

Fig. 2. The denaturation enthalpies plotted vs. corresponding transition temperatures. Markers for the MeCN series are the same as in Fig. 1. The lines are best fit results and drawn for the constant MeCN concentrations: solid line, $x_{3}=0$; long dash, 0.037 ; short dash, 0.075 .

The mechanism of unfolding transitions was close to 'all-or-none' as reflected by the values of the van't Hoff parameter $\Delta(\mathrm{vh} / \Delta$ (cal [17] presented in Fig. 3. The two-state character of unfolding transitions allowed calculations of the unfolding enthalpy and entropy changes, Gibbs free energy of protein structure stabilization and other derivative parameters such as proton uptake and preferential solvation changes upon unfolding. For the evaluation details see Appendix B. 


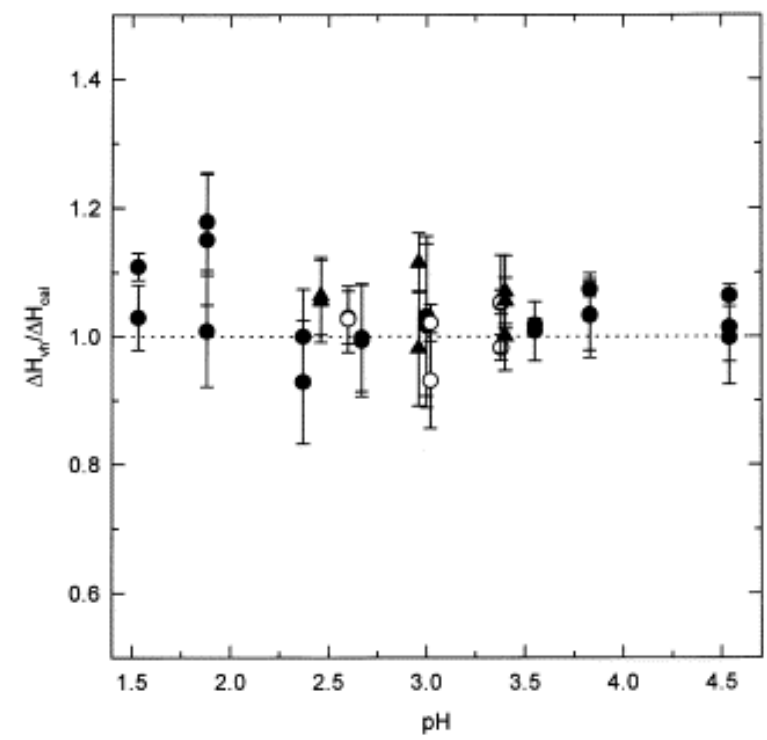

Fig. 3. The van't Hoff parameter values calculated for all experimental transitions and plotted vs. the $\mathrm{pH}$ value of the solution. Markers are the same as in Fig. 1 and Fig. 2.

\section{Discussion}

\subsection{Enthalpy of unfolding}

Fig. 4 shows the enthalpy difference between the native and denatured states of lysozyme extrapolated to four temperatures and plotted as a function of the $\mathrm{MeCN}$ mole fraction (the error of extrapolation is shown by error bars). This plot indicates that within the studied $\mathrm{MeCN}$ concentration range the heat absorbed on unfolding grows up on addition of $\mathrm{MeCN}$, and after passing through a maximum tends to decrease.

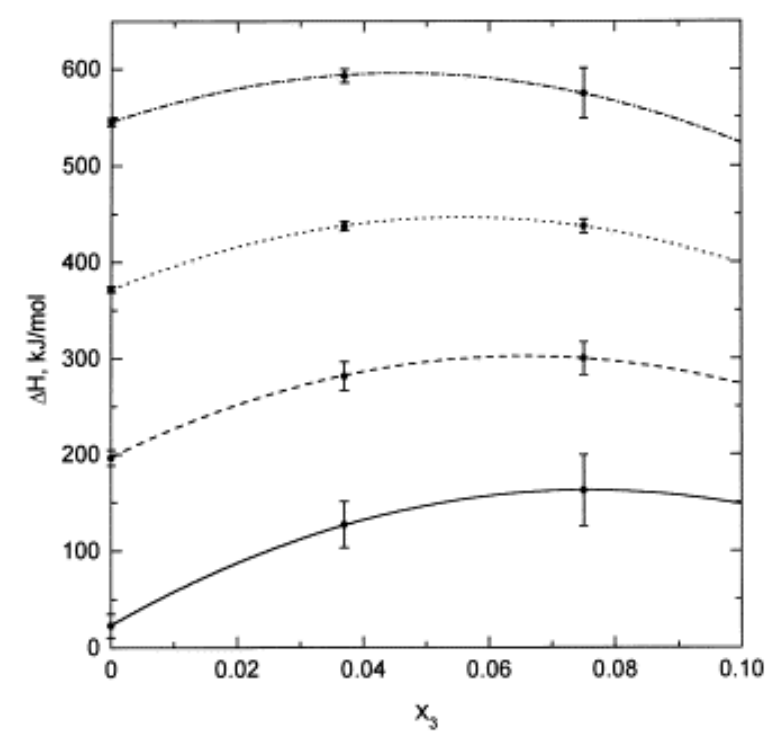

Fig. 4. The enthalpy difference between the native and denatured states of lysozyme vs. the MeCN mole fraction extrapolated to the constant temperatures: solid line, $270 \mathrm{~K}$; long dash, $300 \mathrm{~K}$; short dash, $330 \mathrm{~K}$; dash-dot-dash, $360 \mathrm{~K}$. Symbols stand to indicate the solution compositions at which DSC experiments were carried out. Error bars show the accuracy of extrapolated values. 
According to Murphy and Freire [18], the growth of $\Delta \mathrm{H}$ on addition of an organic solvent can be explained by the assumption that $\mathrm{MeCN}$ diminishes the hydrophobic contribution to the unfolding enthalpy (which is believed to be negative) while the hydrogen bonding contribution (authors argue that this is positive and is the major contribution to the heat of unfolding) remains nearly constant at a low organic solvent concentration. On the other hand, the situation might not be that simple for the hydrophobic part of the unfolding enthalpy which may not be a monotonic decreasing function of the MeCN content. Ben-Naim reported data [19] that the pairwise hydrophobic interaction enthalpy in water-ethanol solutions initially increases and only above some ethanol content begins to decrease; the phenomenon that at different solvent compositions different modes of solvent packing (different mixing schemes) are present in aqueous solutions was thoroughly discussed elsewhere [20].

\subsection{Thermodynamic stability of lysozyme in aqueous solutions of $\mathrm{MeCN}$}

The most attractive feature of thermodynamic analysis is that it allows one to estimate stability of a protein over a range of conditions of interest. It should be noted (following Plaza del Pino and Sanchez-Ruiz [21]) that we speak here about thermodynamic stability which is in effect the work required to disrupt the protein tertiary structure under particular solution conditions and temperature, in contrast to thermal or operational stability which is understood simply as a temperature at which a protein denatures (or inactivates) at a particular solvent composition and $\mathrm{pH}$.

Thermodynamic stability (the stabilization free energy) is directly related to the equilibrium between native and denatured states of the protein. This means that the higher the thermodynamic stability the less the probability for the native protein molecule to unfold due to thermal fluctuations and the less the time interval during which each protein molecule is unfolded. This, in turn, determines sensitivity of the protein to hydrolysis or inactivation due to some chemical modifications or aggregation of unfolded polypeptide chains. Thus, thermodynamic stability (or stabilization free energy) of a protein is a parameter which is directly related to a real lifetime of proteins in solutions under native conditions.

The knowledge of reliable $\Delta C_{p}$ values allowed us to estimate the thermodynamic stability of lysozyme over a range of solution composition and temperature. Details on the free energy calculations are given in Appendix B. Fig. 5 represents the extrapolated values of the free energy of native structure stabilization $\Delta\left(^{\circ}\right.$ as a function of mole fraction of $\mathrm{MeCN}$ at four temperatures and constant $\mathrm{pH}$ of 3.4. A rather small error associated with $\Delta /^{\circ}$ values in the high temperature region is somewhat surprising. One might expect it to be much greater since $\Delta r^{\circ}$ is calculated as a difference between two large terms $\Delta$ (and ( $\Delta$ (. However, in fact, $\Delta$ (is calculated using $\Delta$ (data, therefore, the errors of the $\Delta$ ( and ( $\Delta$ ( terms are highly correlated leading to a significant cancellation of error in the result.

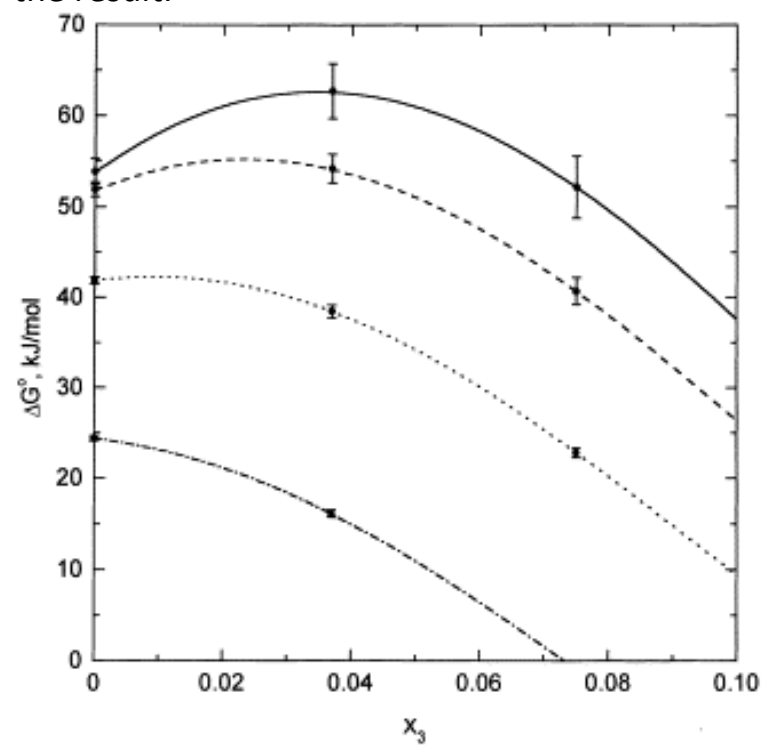


Fig. 5. The free energy difference between the native and denatured state of lysozyme vs. the MeCN mole fraction at pH 3.4 extrapolated to the constant temperatures: solid line, $270 \mathrm{~K}$; long dash, $290 \mathrm{~K}$; short dash, 310 $\mathrm{K}$; dash-dot-dash, $330 \mathrm{~K}$. Symbols stand to indicate the solution compositions at which DSC experiments were carried out. Error bars show the accuracy of extrapolated values.

The most prominent phenomenon seen in Fig. 5 is that the destabilizing effect of MeCN on lysozyme decreases on lowering the temperature (the $\mathrm{pH}$ value of 3.4 showed the most pronounced effect). Moreover, below room temperature our calculations predict stabilization of the protein on addition of MeCN. This finding is in line with the results cited above on stabilizing effect at low temperatures predicted for alcohols [9], [10], 2-methyl-2,4pentanediol and ethylene glycol [7], [11]. All these substances, including MeCN, have significant hydrophobic moiety in the molecule. There is evidence from solution chemistry that at low temperatures and small concentrations of non-ionic additives, hydrophobic interactions in aqueous solutions are increased relatively to those in pure water (i.e.: argon solubility in ethanol and dioxane aqueous solutions [22], [23]). Therefore, the presence of small amounts of such additives would shift folding-unfolding equilibrium to the left, thus stabilizing the folded form of a protein.

Another factor influencing protein-solvent interactions is the presence of charges on the surface of a protein exerting repulsive forces onto molecules of non-aqueous component in the neighborhood of the protein in solution [2]. There is evidence for very low solubility of charged amino acids in aqueous acetonitrile [24] showing strong repulsion of $\mathrm{MeCN}$ molecules from charged groups. If this factor plays an important role in the above stabilizing effect, the latter should strongly depend on the $\mathrm{pH}$ value of the medium. Fig. 6 represents values of the protein stabilization free energy extrapolated to $270 \mathrm{~K}$ and corresponding to minimal and maximal $\mathrm{pH}$ values studied. Our estimates of the proton uptake upon unfolding revealed that at the lowest $\mathrm{pH}(\mathrm{pH} 2.5)$ lysozyme absorbs about 4-5 protons per protein molecule, while at $\mathrm{pH} 3.5$ this value is close to zero (for details see Appendix B Analytical expressions for entropy of unfolding, Gibbs free energy of protein native structure stabilization, proton uptake and preferential solvation changes on unfolding, Appendix C Proton uptake on unfolding). This means that at $\mathrm{pH} 3.5$ the native and denatured forms of lysozyme have an equal charges while at $\mathrm{pH} 2.5$ the denatured lysozyme is charged more positively than the native one. However, as can be judged from Fig. 6 , the stabilization effect at the low temperature hardly feels this difference (at least the difference is less than the extrapolation uncertainty). Therefore, one could draw the conclusion that the charge-induced contribution is not dominant in the stabilization of lysozyme by small amounts of MeCN at low temperatures; an increase of hydrophobic interactions in dilute acetonitrile seems the most likely cause of the stabilization effect.

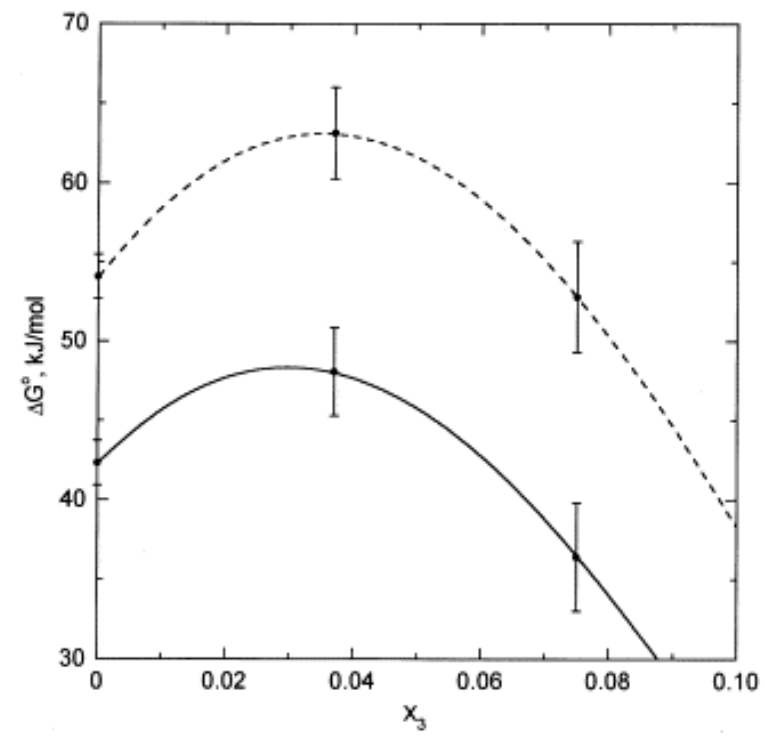


Fig. 6. The free energy difference between native and denatured states of lysozyme extrapolated to $270 \mathrm{~K}$ at two $\mathrm{pH}$ values: solid line, $\mathrm{pH} 2.5$; dash, $\mathrm{pH}$ 3.5. Symbols stand to indicate the solution compositions at which DSC experiments were carried out. Error bars show the accuracy of extrapolated values.

\subsection{Proton uptake on unfolding}

Exposure of anomalous titratable groups on lysozyme unfolding at acid $\mathrm{pH}$ of the medium leads to absorption of protons on transition $\Delta_{l} v[25]$. This quantity was calculated for the constant $\mathrm{pH} 3.0$ and found to be independent of ( and slightly growing on increasing the MeCN content $(2.2 \pm 0.1$ mole protons per mole of a protein at $0 \%$ $\mathrm{MeCN} ; 3.0 \pm 0.2$ at $10 \%$; and $3.3 \pm 0.2$ at $19 \% \mathrm{MeCN})$. This is unlike the situation found by Plaza del Pino and Sanchez-Ruiz [21] for ribonuclease A in sarcosine-water solutions where the $\Delta_{l} v$ values were independent of the solution composition as well as of the temperature. In our case, the growth of $\Delta_{l} v$ was attributed to the increase in $\mathrm{pK}$ values of titratable groups caused by the presence of MeCN (for details see Appendix C).

\subsection{Preferential solvation change}

The knowledge of the stabilization free energy for lysozyme in aqueous MeCN allowed us to calculate a direct measure of the solvent-protein interactions: the difference in preferential solvation of native and denatured states of the protein. The preferential solvation phenomena have been extensively studied in the last decade in a number of theoretical and experimental works (for example [2], and references therein). Preferential solvation is a purely thermodynamic characteristic of the interactions of a macromolecule with solution components. Its value can be measured in a number of ways; denaturational studies appear to be particularly suitable for measuring the change of this quantity for conformational transitions in proteins. The thermodynamic background of this approach was elaborated by Wyman [26], Tanford [27], Schellman [28], [29] and others (for details see Appendix D).

In our earlier paper we demonstrated that the preferential solvation change $\Delta \Gamma$ on protein unfolding could be a useful parameter for rationalization of interrelations between the properties of a solution itself and protein thermodynamics in such a solution [30]. In the present work we determined the accurate values of the denaturational heat capacity increment $\Delta($, and it allowed us to calculate $\Delta \Gamma$ values not only for the temperatures of transitions (as in that previous work) but also for arbitrary temperatures. Thus, we can separate the temperature and solvent contributions to the preferential solvation change of protein unfolding.

Fig. 7 shows the difference between native and denatured lysozyme in preferential solvation by $\operatorname{MeCN}\left(\Delta \Gamma_{23}\right.$, panel a) and by water $\left(\Delta \Gamma_{21}\right.$, panel b), extrapolated to the constant temperatures and plotted versus the MeCN mole fraction. These two measures of preferential solvation are fundamentally linked and in effect carry the same thermodynamic information; moreover, each of them contains contributions from both water and component 3 molecules in the solvent volume over which a protein molecule affects the chemical potential of $\mathrm{MeCN}$ [31]. However, since the concentration of $\mathrm{MeCN}$ in our experiments is low compared to that of water, $\Delta \Gamma_{23}$ can be viewed as the difference in the number of MeCN molecules between such volumes surrounding native and denatured states of the protein (this approximation holds true in dilute solutions of component 3 [28], [29], [31], [32]). In contrast, $\Delta \Gamma_{21}$ cannot be interpreted in such a simple way since the water concentration is high; however, it appears to be more suitable for the analysis because $\Delta \Gamma_{23}$ values grow on increasing $x_{3}$ rather rapidly and reach high values hindering observation of the difference between the curves for various temperatures. Overall changes of $\Delta \Gamma_{21}$ are less dramatic giving an easier-to-see plot. 

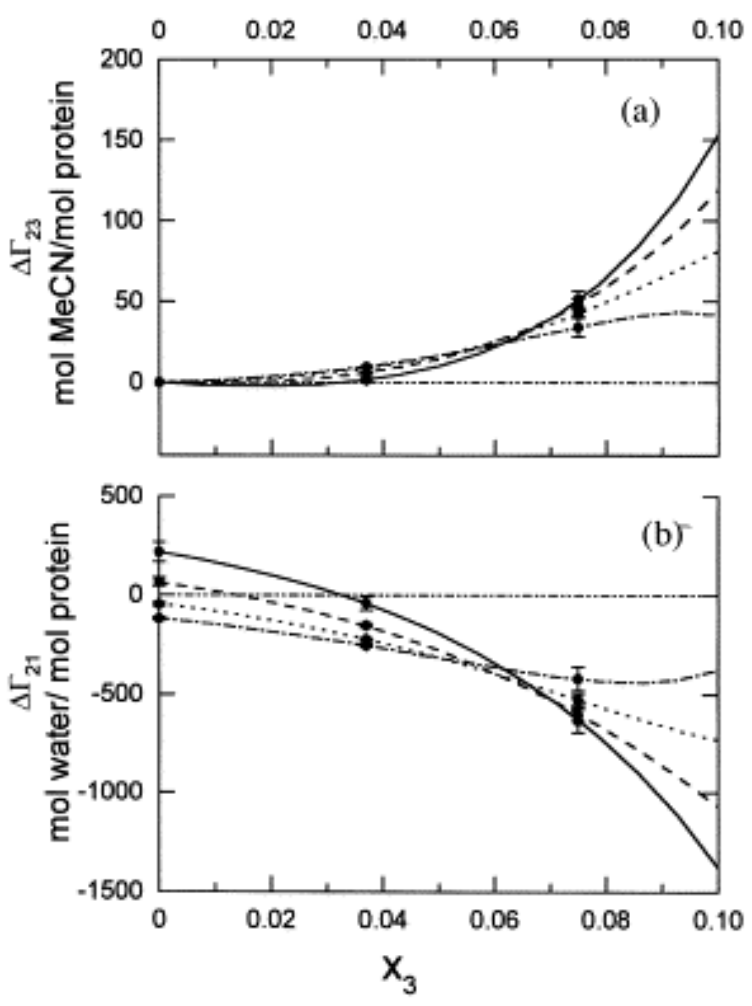

Fig. 7. The difference in the preferential solvation of native and denatured protein by MeCN (panel a) and water (panel b) vs. the MeCN volume fraction. The solvation difference was evaluated for $\mathrm{pH} 3.0$ at the constant temperatures: solid line, $270 \mathrm{~K}$; long dash, $300 \mathrm{~K}$; short dash, $330 \mathrm{~K}$; dash-dot-dash, $360 \mathrm{~K}$. Symbols stand to indicate the solution compositions at which DSC experiments were carried out. Error bars show the accuracy of extrapolated values.

Fig. 7 show that at high temperatures, the values of $\Delta \Gamma_{21}$ are always negative $\left(\Delta \Gamma_{23}-\right.$ positive) indicating that unfolding at these temperatures leads to a state with a larger affinity to component 3 (MeCN) than that of the native state. Lowering of the temperature leads to a decrease in the absolute values of a preferential solvation change; at approximately $300 \mathrm{~K}, \Delta \Gamma_{21}$ reaches zero in the water-rich region implying that at these conditions the total interaction of $\mathrm{MeCN}$ with lysozyme is exactly the same for the native and denatured states. Further lowering of the temperature would change the sign of the solvation difference implying that the native state would be more solvated by MeCN than the denatured state. This is in effect the other side of the same coin, the above hypothetic stabilizing action of $\mathrm{MeCN}$ at low temperatures in the water-rich region.

An interesting phenomenon can be seen at approximately 0.06 mole fractions of $\mathrm{MeCN}$ : the isotherms of preferential solvation change cross here and $\Delta \Gamma$ appears to be independent of temperature over a fairly wide range. This implies that the change in the composition of the protein solvation shell occurring on the unfolding transition does not 'feel' the temperature of the medium at this $\mathrm{MeCN}$ content over at least $60 \mathrm{~K}$.

When analyzing the $\mathrm{pH}$-dependence of the preferential solvation change, we found that $\Delta \Gamma_{21}$ values at $\mathrm{pH} 3.5$ are less negative than at $\mathrm{pH} 2.5$ and this effect turns to the opposite one at about the same 0.06 mole fractions of $\mathrm{MeCN}$ (data not shown).

As mentioned above, the change of the preferential solvation is a purely thermodynamic quantity; it is calculated directly from experimental thermodynamic data with no a priori assumptions. Thus, the abovementioned findings directly characterize equilibrium between native and denatured states of lysozyme in MeCN-water solutions with no regard to the particular model of a molecular mechanism (by definition, $\Delta \Gamma_{2 i}$ is an increment of the unfolding equilibrium constant on increasing the activity of the $i$-th component $\mathrm{d}(\operatorname{In} K) / \mathrm{d}\left(\operatorname{In} a_{i}\right)$ [26], [27]). Nowadays, there are some theoretical approaches developed to describe the protein solvation in 
mixed media (see, for instance: [2], [29], [33], [34], [35]). However, to be applied, they require a number of parameters (in addition to the protein DSC data) to be estimated, such as:

1. equilibrium constants for binding of an organic additive and water to side chains of various amino acid residues and to a peptide backbone;

2. to what extent solvent binding at the neighboring sites is really independent (for example, Schellman's solvent exchange model requires such independence [29]); and

3. how much the structure of the denatured state of the protein changes upon addition of an organic additive - it is well known that high concentrations of additives often cause dramatic changes in the structure of unfolded proteins and peptides (e.g. see [36], [37], [38], [39], [40]).

The latter condition is of great importance because any protein unfolding model deals either with initial and final states of constant structure or they should explicitly take into account that the structure of an unfolded state (as more flexible than the native one) is likely to change on increasing the concentration of a non-aqueous component.

At present time, most of the above mentioned parameters are unavailable for lysozyme in aqueous MeCN. The only data of such a kind are in the paper of Gekko et al. [24] concerning the preferential solvation of native lysozyme in aqueous $\mathrm{MeCN}$ as well as the transfer free energies for amino acid side chains from water to these solutions. Data of such a kind are exactly what are needed for detailed analysis of lysozyme stabilization by small amounts of MeCN in water. However, results of that study cannot be applied here because the lowest concentration point of Gekko's data set is $x_{3}=0.1$ (while our highest MeCN concentration is $x_{3}=0.075$ with the stabilization effect taking place at about 0.03-0.04).

As for preferential solvation, one may still compare the magnitude of effects at the boundary of two data sets. From experiments on equilibrium dialysis, Gekko and co-workers found that native lysozyme at $25^{\circ} \mathrm{C}$ and $x_{3}=0.1$ is strongly preferentially hydrated with $\Gamma_{\mathrm{N} 1} \approx 800 \mathrm{~mol}$ water $/ \mathrm{mol}$ protein. Extrapolation of our $\Delta \Gamma_{21}$ to $x_{3}=0.1$ gives $\approx-1000$ (see Fig. 7). Hence, denatured lysozyme should be preferentially dehydrated with $\Gamma_{\mathrm{D} 1} \approx-200 \mathrm{~mol}$ water/mol protein.

Finally, we would like to emphasize necessity of proper taking into account solution non-ideality when calculating the preferential solvation changes. Fig. 8 shows the same graphs as Fig. 7 but calculations of preferential solvation were done this time assuming that the activity coefficient of MeCN remains constant. One can see (see Fig. 7 and Fig. 8) that in a very dilute region $\left(x_{3}<0.03\right)$ this assumption does not change the result due to the large value of $1 / x_{3}$ term in the denominator which greatly exceeds non-ideality contribution $\partial \ln \left(f_{3}\right) / \partial x_{3}$ [Appendix B, Eq. (B4)]. At the higher MeCN content the calculated values of the preferential solvation change deviate the stronger the higher the $\mathrm{MeCN}$ mole fraction is. This result indicates that it is highly important to take properly into account solution non-ideality when dealing with aqueous solutions at high concentrations of organic additives. 

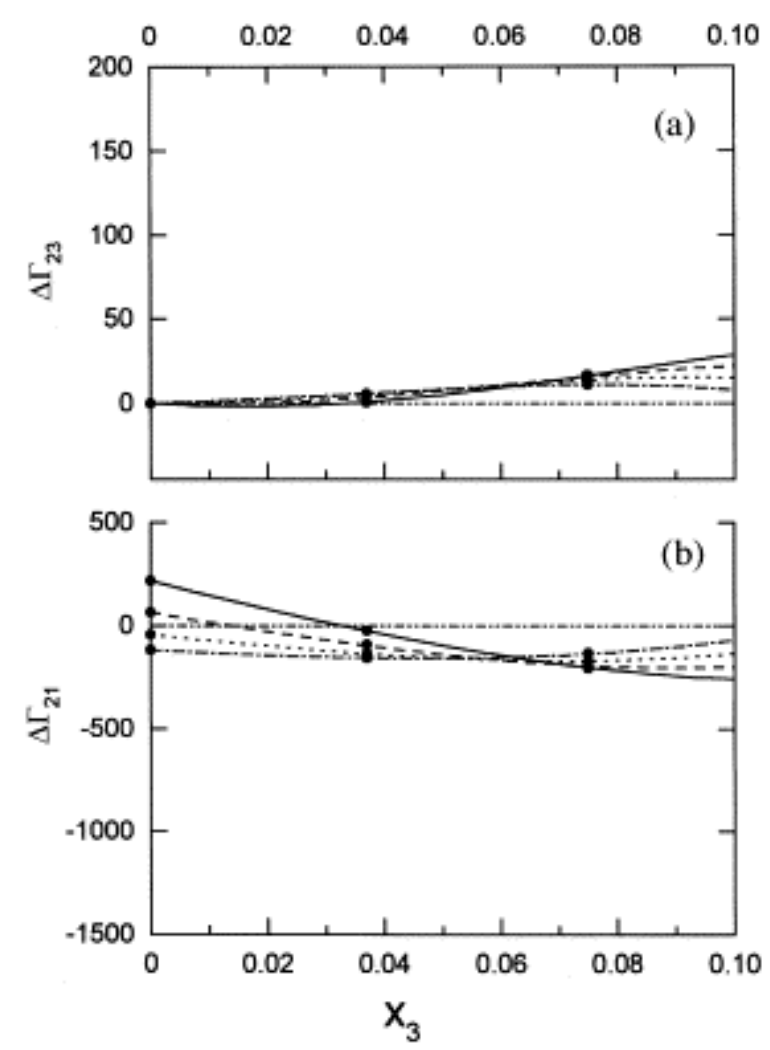

Fig. 8. The same quantities as in Fig. 7 but evaluated this time assuming that the MeCN-water mixture is an ideal solution.

\section{Conclusions}

The phenomenon of increasing protein thermodynamic stability in aqueous solutions by small amounts of denaturing additives was investigated in detail with lysozyme-acetonitrile-water system as an example by means of scanning microcalorimetry. The heat capacity change on protein unfolding transitions was determined with the highest achievable precision making it possible to calculate the free energy of protein stabilization at low temperatures as well as the corresponding preferential solvation changes. The main findings of this study are as follows.

1. MeCN is a 'denaturant' under all conditions studied - its presence diminishes the temperature of lysozyme heat denaturation.

2. Examination of the free energy of protein stabilization calculated from the DSC data revealed that small concentrations of $\mathrm{MeCN}$ should increase thermodynamic stability of lysozyme below room temperature in contrast to the destabilizing effect of $\mathrm{MeCN}$ at higher concentrations and temperatures.

3. The stabilization phenomenon appears to be $\mathrm{pH}$-independent indicating thus that the charge-induced contribution to the stabilization of lysozyme is relatively small.

4. At low temperatures and small concentrations of MeCN the denatured state of lysozyme was predicted to be more hydrated than the native one which accounts for a rise in the protein stabilization free energy.

5. The temperature dependence of the preferential solvation difference between native and denatured states was found to be significant at low concentrations of $\mathrm{MeCN}$ and vanishing on further addition of the co-solvent. When the MeCN mole fraction is about $0.06, \Delta \Gamma$ appears to be independent of the temperature over a range of at least $60 \mathrm{~K}$.

6. Importance of proper taking into account non-ideal properties of mixed solutions, when analyzing preferential solvation is emphasized. 


\section{Acknowledgements}

We are grateful to Dr Peter Westh for critical reading of the manuscript. This study was supported in part by the RFBR-INTAS grant IR-97-1899.

\section{Appendix A. Fitting of the temperature and enthalpy data}

\section{A.1. Temperature of transition}

For further evaluations we found the best fit of the $l_{(}\left(x_{3},()\right.$ data by the polynomial equation:

(A1)

$$
\mathrm{T}_{\mathrm{d}} \mathrm{x}, \mathrm{pH}=\mathrm{t}_{1}+\mathrm{t}_{2} \mathrm{pH}-\mathrm{pH}_{0}+\mathrm{t}_{3} \mathrm{pH}-\mathrm{pH}_{0}{ }^{2}+\mathrm{t}_{4} \mathrm{pH}-\mathrm{pH}_{0}{ }^{3}+\mathrm{t}_{5} \mathrm{pH}-\mathrm{pH}_{0}{ }^{4}+\mathrm{t}_{6} \mathrm{x}-\mathrm{x}_{0}+\mathrm{t}_{7} \mathrm{x}-\mathrm{x}_{0}{ }^{2}+\mathrm{t}_{8} \mathrm{x}-\mathrm{x}_{0} \mathrm{pH}-\mathrm{pH}_{0}
$$

The equation of the fourth order was chosen to reflect the fast decrease in the slope of the ( $v s$. ( curve and had no theoretical significance; $t_{1}, \ldots, t_{8}$ are the parameters to fit; $x$ is the MeCN volume fraction, $x_{3} ;\left(l_{0}, x_{0}\right.$ were chosen near the center of the area covered. This polynomial equation simulates well the temperature of transitions within the $\mathrm{pH}$ range 2.1-3.5 (this was the region of our interest).

\section{A.2. Enthalpy of transition}

The dependence of the enthalpy on the temperature was taken to be linear (this assumption would not introduce large error into calculated values of stabilization free energy, at least, as the first approximation; see [41], [42]). The (( contribution to the heat of unfolding was believed to be negligible in this acid region, since heats of titration under acid conditions are rather small [25] and the compensating buffer substance (glycine) was present in the medium. The heat capacity change was also assumed to be independent of $(($, so we chose the following equation to fit the enthalpy data:

$$
\Delta \mathrm{Hx}, \mathrm{T}=\Delta \mathrm{H}_{0} \mathrm{x}+\Delta \mathrm{C}_{\mathrm{p}} \mathrm{x}^{*} \mathrm{~T}-\mathrm{T}_{0}
$$

where

$$
\begin{gathered}
\Delta \mathrm{H}_{0} \mathrm{x}=\mathrm{h}_{1}+\mathrm{h}_{2} \mathrm{x}-\mathrm{x}_{0}+\mathrm{h}_{3} \mathrm{x}-\mathrm{x}_{0}{ }^{2}, \\
\Delta \mathrm{C}_{\mathrm{p}} \mathrm{x}=\mathrm{h}_{4}+\mathrm{h}_{5} \mathrm{x}-\mathrm{x}_{0},
\end{gathered}
$$

$h_{1}, \ldots, h_{5}$ are the parameters to fit; $x$ is the MeCN mole fraction, $x_{3} ; x_{0}$, (o are the center of the conditions area.

\section{Appendix B. Analytical expressions for entropy of unfolding, Gibbs free energy of protein native structure stabilization, proton uptake and preferential solvation changes on unfolding}

Having fitted the analytical equations to the experimental enthalpy and temperature data we can calculate all the thermodynamic quantities of protein structure stabilization in $\mathrm{MeCN}-$ water solutions as functions of (, / ( and $x_{3}$ using the following relations:

\section{B.1. Entropy change}

$$
\Delta S x_{3}, T, p H=\Delta H x_{3}, T_{d} x_{3}, p H / T_{d} x_{3}, p H+\Delta C_{p} x_{3}{ }^{*} \ln T / T_{d} x_{3}, p H .
$$


B.2. Standard free energy of stabilization:

$$
\Delta \mathrm{G}^{\mathrm{o}} \mathrm{x}_{3}, \mathrm{~T}, \mathrm{pH}=\Delta \mathrm{H} \mathrm{x}_{3}, \mathrm{~T}-\mathrm{T}^{*} \Delta \mathrm{S} \mathrm{x}_{3}, \mathrm{~T}, \mathrm{pH} .
$$

\section{B.3. Proton uptake upon unfolding}

$$
\Delta_{\mathrm{d}} \mathrm{vx}_{3}, \mathrm{~T}, \mathrm{pH}=\partial \Delta \mathrm{G}^{\mathrm{o}} \mathrm{x}_{3}, \mathrm{~T}, \mathrm{pH} \partial \mathrm{pH} \mathrm{T}_{\mathrm{x} 3}{ }^{*} \ln 10 * \mathrm{RT}^{-1} \text {, }
$$

where $R=8.31 \mathrm{~J} / \mathrm{mol}^{*} \mathrm{~K}$. The derivative of $\Delta\left(^{\circ}\right.$ with respect to ( can be calculated either numerically or analytically.

\section{B.4. Preferential solvation change upon unfolding:}

$$
\Delta \Gamma_{23} x_{3}, T, p H=-\partial \Delta G^{o} x_{3}, T, p H / \partial x_{3}{ }_{T, p H} / R T \partial I n a_{3} / \partial x_{3}{ }_{T, p H}=-\partial \Delta G^{o} x_{3}, T, p H / \partial x_{3}{ }_{T, p H} / R T 1 / x_{3}-\partial \operatorname{lnf}_{3} / \partial x_{3}{ }_{T, p H}
$$

where $x$ is $x_{3} ; a_{3}$ is the MeCN activity in solution and $f_{3}$ is the corresponding activity coefficient of MeCN on the mole fraction scale. For details on the evaluation of solvation changes for protein denaturation see Appendix $\mathrm{D}$.

\section{Appendix C. Proton uptake on unfolding}

The proper interpretation of the changes in a number of protons bound/released upon unfolding requires knowledge of the ionization constants of the amino acid side chains under conditions of interest. No data of such a kind were available for MeCN-water mixtures. However, the assumption that their pK values grow up on increasing the MeCN content can be supported by the evidence that the pK values of such substances as phenol, acetic, propionic and benzoic acids (somewhat mimicking the protein $\alpha-\mathrm{COOH}, \mathrm{Glu}$ and Asp side chains) increase by approximately $2 \mathrm{pK}$ units on addition of ethanol up to $70 \%$ in water solutions and by 3-3.5 pK units on addition of acetone up to the same high concentration [43], [44]. Therefore, an increase in pK values of the protein titratable groups in $\mathrm{MeCN}$-water mixtures can also be expected since $\mathrm{MeCN}$ does not differ much from acetone and ethanol by physico-chemical properties.

\section{Appendix D. The preferential solvation change upon protein denaturation}

Following Schellman [29], we define preferential solvation by the quantity $\Gamma_{23}$, which is read as 'the preferential solvation of component 2 by $3^{\prime}$ or as 'the selective interaction of component 3 with 2':

$$
\Gamma_{23} \equiv \partial v_{3} / \partial v_{2}
$$

at the constant ( and $\mu_{3}$. This quantity represents the number of molecules of $3, v_{3}$, that must be added to the solution on addition of one macromolecule, 2 , to keep the chemical potential of component $3, \mu_{3}$ constant. On the other hand, this quantity reflects directly the change of the chemical potential of a macromolecule, $\mu_{l}$, on addition of component 3 :

$$
\Gamma_{23}=-\partial \mu_{2} / \partial \mu_{3}, T, x_{2}=\text { const, }
$$

where $\mu_{3}$ is the chemical potential of component $3 ; x_{2}$ is the macromolecule mole fraction. The quantity $\Gamma_{21}$ reflecting the selective interaction with water can be derived in a fully symmetric fashion and has a fundamental relation to $\Gamma_{23}[31]$ by 


$$
\Gamma_{21} / \Gamma_{23}=-x_{1} / x_{3}
$$

By definition preferential solvation depends on the nature of the solvated surface, so its value is conformationdependent for macromolecules. Therefore, we may define the denaturational change of preferential solvation $\Delta \Gamma_{23}$ of protein by component 3 as the difference between preferential solvation of denatured and native states:

$$
\Delta \Gamma_{23}=\Gamma_{\mathrm{D} 3}-\Gamma_{\mathrm{N} 3}
$$

When the concentration of component 3 is low, $\Delta \Gamma_{23}$ represents a number of component 3 molecules entering or leaving the solvent volume over which a protein molecule affects the chemical potential of component 3 (one should note that $\Delta \Gamma_{21}$ does not have such a straightforward interpretation since the water concentration usually is very high [29], [31]). $\Delta \Gamma_{23}$ can be readily found for protein denaturation as

$$
\Delta \Gamma_{23}=-\partial \Delta G^{o} / \partial x_{3, p H, x} \partial \mu_{3} / \partial x_{3 T, p H, x} \cdot
$$

The numerator of Eq. (D5) contains $\partial \Delta \nu^{\circ} / \partial\left(\right.$, where $\Delta \gamma^{\circ}$ is the Gibbs free energy difference between the native and denatured states. Since we have $\Delta\left(^{\circ}\right.$ expressed in the analytical form (see Appendix B) we can calculate it for any conditions. $\partial \mu_{3} / \partial x_{3}$ in the denominator reflects the non-ideality of the water-organic solution and, on condition $x_{2} \rightarrow 0$, can be evaluated on the basis of the partial vapour pressures above binary water-organic mixtures. The latter approach is well known and can be found elsewhere (for instance, see [45], [46]). The requirement of constant $\mathrm{pH}$ in the denominator may be disregarded in our particular case since MeCN doesn't have ionizable groups which make its activity insensible to moderate $\mathrm{pH}$ changes. The MeCN activity data were taken from and critically examined on self-consistency as recommended by Kogan et al. [45]. The temperature effect on MeCN activity was found to be negligible by comparing the data for $20^{\circ} \mathrm{C}$ and $30^{\circ} \mathrm{C}$.

\section{References}

[1] T. Arakawa, J.F. Carpenter, Y. Kita, J.H. Crowe The basis for toxicity of certain cryoprotectants: a hypothesis Cryobiology, 27 (1990), pp. 401-415

[2] S.N. Timasheff The control of protein stability and association by weak interactions with water: how do solvents affect these processes Annu. Rev. Biophys. Biomol. Struct., 22 (1993), pp. 67-97

[3] K. Griebenow, A.M. Klibanov On protein denaturation in aqueous-organic mixtures but not in pure organic solvents J. Am. Chem. Soc., 118 (1996), pp. 1170-11695

[4] S.Y. Gerlsma Reversible denaturation of ribonuclease in aqueous solutions as influenced by polyhydric alcohols and some other additives J. Biol. Chem., 243 (1968), pp. 957-961

[5] S.Y. Gerlsma The effects of polyhydric and monohydric alcohols on the heat induced reversible denaturation of chymotrypsinogen A Eur. J. Biochem., 14 (1970), pp. 150-153

[6] S.Y. Gerlsma, E.R. Stuur The effect of polyhydric and monohydric alcohols on the heat-induced reversible denaturation of lysozyme and ribonuclease Int. J. Pept. Protein Res., 4 (1972), pp. 377-383

[7] Y. Fujita, Y. Noda The effect of ethylene glycol on the thermal denaturation of ribonuclease $A$ and chymotrypsinogen A as measured by differential scanning calorimetry Bull. Chem. Soc. Jpn., 57 (1984), pp. 2177-2183

[8] R. Skukuya, G.J. Schwert Glutamic acid decarboxylase. III. The inactivation of enzyme at low temperatures Biol.Chem., 235 (1960), p. 1658

[9] J.F. Brandts, L. Hunt The thermodynamics of protein denaturation. III. The denaturation of ribonuclease in water and in aqueous urea and ethanol mixtures J. Am. Chem. Soc., 89 (1967), pp. 4826-4838[10]

G. Velicelebi, J.M. Sturtevant Thermodynamics of the denaturation of lysozyme in alcohol-water mixtures Biochemistry, 18 (1979), pp. 1180-1186 
[11] Y. Fujita, Y. Noda Differential scanning calorimetric studies on the thermal denaturation of ribonuclease A in aqueous 2-methyl-2,4-pentanediol Bull. Chem. Soc. Jpn., 57 (1984), pp. 1891-1896

[12] N.A. Izmailov Solution Electrochemistry in Russian, 2nd ed., Chemistry Publ, Moscow (1966)

[13] E.P. Serjeant. Potentiometry and Potentiometric Titrations. In: Chemical Analysis, vol. 69, John Wiley \& Sons, New York, 1984.

[14] M.L. Johnson, L.M. Faunt Parameter estimation by least-squares methods Methods Enzymol., 210 (1992), pp. 1-37

[15] M. Straume, M.L. Johnson Monte-Carlo method for determining complete confidence probability distributions of estimated model parameters Methods Enzymol., 210 (1992), pp. 117-129

[16] P.L. Privalov, N.N. Khechinashvili A thermodynamic approach to the problem of stabilization of globular protein structure: a calorimetric study J. Mol. Biol., 86 (1974), pp. 665-684

[17] P.L. Privalov, S.A. Potekhin Scanning microcalorimetry in studying temperature-induced changes in proteins Methods Enzymol., 131L (1986), pp. 4-51

[18] K.P. Murphy, E. Freire Thermodynamics of structural stability and cooperative folding behavior in proteins Adv. Protein Chem., 43 (1992), pp. 313-361

[19] A. Ben-Naim Hydrophobic Interactions, Plenum Press, New York (1978)

[20] Y. Koga Mixing schemes in aqueous solutions of non-electrolytes: a thermodynamic approach J. Phys. Chem., 100 (1996), pp. 5172-5181

[21] I.M. Plaza del Pino, J.M. Sanchez-Ruiz An osmolyte effect on the heat capacity change for protein folding Biochemistry, 34 (1995), pp. 8621-8630[22]

A. Ben-Naim, S. Baer Solubility and thermodynamics of solution of argon in the water-ethanol system Trans. Faraday Soc., 60 (1964), pp. 1736-1741

[23] A. Ben-Naim, G. Moran Solubility and thermodynamics of solution of argon in the water+p-dioxane system Trans. Faraday Soc., 61 (1965), pp. 821-825

[24] K. Gekko, E. Ohmae, K. Kameyama, T. Takagi Acetonitrile-protein interactions: amino acid solubility and preferential solvation Biochim. Biophys. Acta., 1387 (1998), pp. 195-205

[25] W. Pfeil, P.L. Privalov Thermodynamic investigations of proteins. III. Thermodynamic description of lysozyme Biophys. Chem., 4 (1976), pp. 41-50

[26] J. Wyman Linked functions and reciprocal effects in hemoglobin: a second look Adv. Protein Chem., 19 (1964), pp. 223-286

[27] C. Tanford Extension of the theory of linked functions to incorporate the effects of protein hydration J. Mol. Biol., 39 (1969), pp. 539-544

[28] J.A. Schellman Solvent denaturation Biopolymers, 17 (1978), pp. 1305-1322

[29] J.A. Schellman A simple model for solvation in mixed solvents. Application to the stabilization and destabilization of macromolecular structures Biophys. Chem., 37 (1990), pp. 121-140

[30] E.L. Kovrigin, S.A. Potekhin Preferential solvation changes upon lysozyme heat denaturation in mixed solvents Biochemistry, 36 (1997), pp. 9195-9199

[31] E.F. Casassa, H. Eisenberg Thermodynamic analysis of multicomponent solutions Adv. Protein Chem., 19 (1964), pp. 287-395

[32] H.O. Hammou, I.M. Plaza del Pino, J.M. Sanchez-Ruiz. Hydration changes upon protein folding: cosolvent effect analysis, New J. Chem. (1998) 1453-1461.

[33] E. Reisler, Y. Haik, H. Eisenberg Bovine serum albumin and aqueous guanidine hydrochloride solutions. Preferential and absolute interactions and comparison with other systems Biochemistry, 16 (1977), pp. 197-203

[34] H. Eisenberg Biological Macromolecules and Polyelectrolytes in Solutions, Clarendon, Oxford (1976)

[35] H. Eisenberg Protein and nucleic acid hydration and cosolvent interactions: establishment of reliable baseline values at high cosolvent concentrations Biophys. Chem., 53 (1994), pp. 57-68

[36] N. Hirota, K. Mizuno, Y. Goto Cooperative $\alpha$-helix formation of $\beta$-lactoglobulin and mellitin induced by hexafluoroisopropanol Protein Sci., 6 (1997), pp. 416-421 
[37] K. Shiraki, K. Nishikawa, Y. Goto Trifluoroethanol-induced stabilization of the alpha-helical structure of beta-lactoglobulin: implication for non-hierarchical protein folding J. Mol. Biol., 245 (1995), pp. 180194

[38] A. Alexandrescu, Y-L. Ng, C.M. Dobson Characterization of a trifluoroethanol-induced partially folded state of alpha-lactalbumin J. Mol. Biol., 235 (1994), pp. 587-599

[39] Y.O. Kamatari, T. Konno, M. Kataoka, K. Akasaka The methanol-induced transition and the expanded helical conformation in hen lysozyme J. Mol. Biol., 259 (1996), p. 512

[40] K.A. Bolin, M. Pitkeathly, A. Miranker, L.J. Smith, C.M. Dobson Insight into a random coil conformation and an isolated helix: structural and dynamical characterisation of the C-helix peptide from hen lysozyme J. Mol. Biol., 261 (1996), pp. 443-453

[41] P.L. Privalov Thermodynamic problems of protein structure Annu. Rev. Biophys. Biophys. Chem., 18 (1989), pp. 47-69

[42] W. Pfeil Protein Stability and Folding: a Collection of Thermodynamic Data, Springer-Verlag, Berlin, Heidelberg, New York (1998)

[43] J.O. Frohliger, R.A. Gartska, H.W. Irwin, O.W. Steward Determination of ionization constants of monobasic acids in ethanol-water solvents by direct potentiometry Anal. Chem., 40 (1968), pp. 1408-1411

[44] C.C. Panichajakul, E.M. Wooley Potentiometric method for determination of acid ionization constants in aqueous organic mixtures Anal. Chem., 47 (1975), pp. 1860-1863

[45] V.B. Kogan, V.M. Fridman, V.V. Kafarov Liquid-Vapour Equilibria (in Russian), Nauka, Moscow (1966)

[46] J.T.W. Lai, F.W. Lan, D. Robb, et al. Excess partial molar enthalpies, entropies, gibbs energies, and volumes in aqueous dimethylsulfoxide J. Solution Chem., 24 (1995), pp. 89-102 